\title{
SIGNIFICADO DE SER CUIDADOR DE PESSOA COM OXIGENOTERAPIA DOMICILIAR: GROUNDED THEORY
}

\section{MEANINGOFBEINGACAREGIVEROFAPERSONWITHHOMEOXYGENTHERAPY: GROUNDED THEORY}

\section{SIGNIFICADO DE SER UN CUIDADOR DE PERSONA CON TERAPIA DE OXÍGENO EN CASA: GROUNDED THEORY}

Oyara de Castro ${ }^{1}$, Rogério Silva Lima ${ }^{2}$, Roberta Seron Sanches ${ }^{3}$, Eliza Maria Rezende Dázio ${ }^{4}$, Roberta Garcia Gomes ${ }^{5}$, Silvana Maria Coelho Leite Fava 6

\section{RESUMO}

Objetivo: compreender o significado de ser cuidador familiar de pessoa com doença pulmonar obstrutiva crônica (DPOC) dependente de oxigenoterapia domiciliar. Método: pesquisa qualitativa fundamentada nos conceitos e pressupostos do Interacionismo Simbólico e da Teoria Fundamentada nos Dados, desenvolvida em domicílio de 20 cuidadores familiares de um município ao Sul de Minas Gerais, por meio de entrevista, observação participante e elaboração de memorando. Resultados: foram construídas as categorias "Se descobrindo como cuidador" e "Mudando a situação de vida em função do papel de cuidador" que resultou deste processo o modelo teórico Ressignificando a vida e o papel de cuidador. Dadas as modificações na vida do cuidador e as atribuições inerentes a essa função, a pessoa busca por novos significados que lhe permitem dar um novo sentido à sua existência. Conclusões: o modelo teórico construído representa o significado de ser cuidador familiar de pessoa com DPOC dependente de oxigenoterapia domiciliar sob categoria central que integrou todas as outras categorias. Este modelo permitiu um entendimento amplo do fenômeno estudado, com base nas perspectivas do próprio cuidador e no contexto no qual ele se insere. Descritores: Cuidadores; Pneumopatias; Oxigenoterapia; Assistência domiciliar; Enfermagem.

\section{ABSTRACT}

Objective: to understand the meaning of being a family caregiver of a person with chronic obstructive pulmonary disease dependent on home oxygen therapy. Method: Qualitative research based on the concepts and assumptions of Symbolic Interactionism and Data-Based Theory, developed at the home of 20 family caregivers in a municipality in southern Minas Gerais, Brazil,using interviews, participant observation and drafting of a memorandum. Results: From the analysis, the categories "Discovering yourself as a caregiver" and "Changing the situation of life according to the role of caregiver" that resulted from this process the theoretical model signifying life and the role of caregiver. Given the changes in the caregiver's life and the attributions inherent to this role, the person seeks new meanings that allow him or her to give a new meaning to his existence. Conclusions: The constructed theoretical model represents the meaning of being a family caregiver of a person coping with chronic obstructive pulmonary disease (COPD) dependent on home oxygen therapy under a central category that integrated all other categories. This model allowed a broad understanding of the studied phenomenon from the perspectives of the caregiver and in the context in which he or she is inserted.

Descriptors:Caregivers; Lung Diseases; Oxygen Inhalation Therapy; Home Nursing; Nursing.

\section{RESUMEN}

Objetivo: Entender el significado de ser un cuidador familiar de una persona con enfermedad pulmonar obstructiva crónica dependiente de la oxigenoterapia en el hogar. Método: Investigación cualitativa basada en los conceptos y supuestos del Interaccionismo Simbólico y Teoría Basada en Datos, desarrollado en el hogar de 20 cuidadores familiares en un municipio al sur de Minas Gerais, a través de entrevistas, observación de los participantes y redacción de un memorándum. Resultados: A partir del análisis, las categorías "Descubrirse como cuidador" y "Cambiar la situación de la vida según el papel del cuidador" que resultaron de este proceso el modelo teórico "Resignificante de la vida y el papel del cuidador". Discusión: Dados los cambios en la vida del cuidador y las atribuciones inherentes a este papel, la persona busca nuevos significados que le permitan dar un nuevo significado a su existencia. Conclusiones: El modelo teórico construido representa el significado de ser un cuidador familiar de una persona con EPOC dependiente de la oxigenoterapia casera bajo una categoría central que integró todas las demás categorías. El modelo permitió una amplia comprensión del fenómeno estudiado desde las perspectivas del propio cuidador y en el contexto en el que se inserta.

Descriptores: Cuidadores; Enfermedades Pulmonares; Terapia por Inhalación de Oxígeno; Atención Domiciliaria de Salud; Enfermería.

${ }^{1}$ Mestre em Enfermagem. Docente da Escola de Enfermagem Wenceslau Brás de Itajubá. ${ }^{2}$ Doutor. Docente da Escola de Enfermagem da Universidade Federal de Alfenas-MG. ${ }^{3}$ Doutor. Docente da Escola de Enfermagem e do Programa de Pós-graduação em Enfermagem da Universidade Federal de Alfenas-MG. ${ }^{4}$ Doutora. Docente da Escola de Enfermagem e do Programa de Pós-graduação em Enfermagem da Universidade Federal de Alfenas-MG. ${ }^{5}$ Mestre em Enfermagem. Enfermeira da Escola de Enfermagem da Universidade Federal de Alfenas-MG. ${ }^{6}$ Doutora. Docente da Escola de Enfermagem e do Programa de Pós-graduação em Enfermagem da Universidade Federal de Alfenas-MG

Como citar este artigo:

Castro O, Lima RS, Sanches RS, et al .et al. Significado de ser cuidador de pessoa com oxigenoterapia domiciliar : Grounded Theory. Revista de Enfermagem do Centro Oeste Mineiro 2020;10:e3607. [Accesss_] Available in http//doi.org/10.19175/recom.v10i0.3607 


\section{INTRODUÇÃO}

A Doença Pulmonar Obstrutiva Crônica (DPOC) é progressiva e com repercussões sistêmicas ${ }^{(1)}$. As pessoas que vivem com essa enfermidade podem apresentar diferentes graus de dependência por complicações e limitações que Ihes são impostas pelo processo de adoecimento. A dispneia, um sintoma cardinal desta morbidade, impõe ao portador de DPOC modificações no estilo de vida, limitações nas atividades de lazer e perda das atividades laborais ${ }^{(2)}$. Desta forma, em virtude das limitações, nas atividades da vida diária, as pessoas com DPOC precisarão, em algum momento de suas trajetórias, da assistência de seus cuidadores informais, a exemplo dos familiares ${ }^{(3)}$.

Neste contexto, os cuidadores informais que, em geral, são pessoas da família, encarregam-se dos cuidados, sem treinamento, remuneração ou vínculo trabalhista ${ }^{(4)}$. Além disso, sua vida é, também, influenciada e modificada ${ }^{(2)}$. Os cuidadores podem se sentir sobrecarregados, ao priorizarem as necessidades de seu familiar doente e relegar a um segundo plano as suas próprias, inclusive, sua saúde. Não raro, podem apresentar comorbidades como obesidade, hipertensão, DPOC não diagnosticada, além de sintomas de ansiedade e fadiga ${ }^{(3)}$.

Este panorama reflete a importância de os profissionais de saúde ampliarem o olhar às urgências dos cuidadores informais de pessoas com DPOC. Entende-se que, para que seja possível propor ações de cuidado ampliadas, que, de fato, atendam às suas demandas, é necessário compreender as interações que os cuidadores estabelecem no cotidiano do cuidar.

Neste sentido, estudos que privilegiem as interações, como aspectos centrais da prática do enfermeiro e favoreçam a produção de novos conhecimentos $^{(5)}$, podem contribuir para dar voz aos cuidadores.

Neste cenário, questiona-se: que é para o cuidador informal ser responsável pelo cuidado de um indivíduo com DPOC em uso de oxigenoterapia em domicílio?

0 objetivo da investigação foi compreender os significados da experiência de ser cuidador informal de pessoa com DPOC em uso de oxigenoterapia em domicílio.

\section{MÉTODO}

Estudo analítico, com abordagem qualitativa, fundamentado no Interacionismo Simbólico ${ }^{(6)}$ E, para o referencial metodológico, elegeu-se a Teoria Fundamentada nos Dados $(\text { TFD })^{(7)}$.

Entende-se que os pressupostos do Interacionismo Simbólico podem contribuir, para a melhor compreensão do fenômeno, uma vez que $\mathrm{o}$ ato de cuidar do familiar é um processo dinâmico, modifica-se continuamente, à medida que $\mathrm{o}$ cuidador interage com $\mathrm{o}$ adoecido $\mathrm{e}$ consigo mesmo e, então, atribui significados às suas ações ${ }^{(6,8)}$.

Pressupõe-se que as ações do cuidador são significativas tanto para ele, quanto para o seu familiar, estabelecendo, dessa forma, as condições possíveis a uma interação simbólica, produtora de ações e comportamentos que apenas podem ser acessados, a partir de uma análise que não desconsidere o processo de construção compartilhada de significados.

Os participantes foram selecionados por conveniência. Buscaram-se, pelo cadastro, pessoas com DPOC, inscritas no Serviço de Oxigenoterapia Domiciliar (SOD) de um município de Minas Gerais, que atendiam aos seguintes critérios: ser cuidador familiar de pessoa com DPOC dependente de oxigenoterapia, exercer a função de cuidador familiar, há pelo menos seis meses, ser residente do município; idade igual ou superior a 18 anos. Dos 25 cuidadores cadastrados, 20 participaram deste estudo, dois se recusaram e três não atenderam aos critérios de elegilibilidade.

O critério que o cuidador exercesse esta função, há pelo menos seis meses, foi estabelecido, em razão de que toda a pessoa necessita de um período mínimo, para conseguir testemunhar uma situação vivenciada ${ }^{(9)}$.

Os dados foram coletados, em 2016, em domicilio, por meio de entrevistas abertas, préagendadas e gravadas em áudio, observação participante e a elaboração de memorandos, elementos da TFD ${ }^{(7)}$.

A abordagem dos participantes ocorreu na própria residência e, nesse primeiro contato, foi explicado o objetivo desta pesquisa, bem como realizado o convite à participação. Ressalta-se que os próprios membros da família, na primeira entrevista, indicaram quem dedicava o maior tempo aos cuidados com o adoecido.

Foram realizadas, em média, três entrevistas com cada participante com duração de 30 a 60 minutos, número suficiente para atingir a saturação teórica e a construção do modelo teórico, conforme determina a TFD ${ }^{(7)}$. As entrevistas foram realizadas pela pesquisadora 
principal que estimou, no momento da entrevista, apenas a presença da pesquisadora e do participante. Utilizou-se um roteiro com questões sobre as características sociais e culturais dos participantes: nome, idade, escolaridade, crença religiosa, ocupação atual e anterior, renda familiar e pessoal, estado marital, número de filhos, grau de parentesco com a pessoa adoecida e tempo em que exerce a função de cuidador. Constituiu-se a questão norteadora: como é para você ser cuidador de uma pessoa com doença pulmonar que tem necessidade de usar oxigênio em domicílio? À medida que os dados foram transcritos e analisados, novos questionamentos foram acrescentados com o objetivo de compreender o fenômeno ${ }^{(7)}$. Os dados foram analisados pelas pesquisadoras com experiência no referencial adotado, concomitantemente à coleta e obedeceram às três etapas da codificação. Na codificação aberta, os dados foram transcritos, examinadas palavras e frases e realizada a extração dos primeiros códigos. Iniciou-se o processo de comparação dos dados, a fim de buscar similaridades e diferenças conceituais que constituíram as categorias temporárias. $\mathrm{Na}$ sequência, realizou-se a codificação axial, em que foi estabelecida a relação entre categorias e suas subcategorias, para maior aproximação com o fenômeno estudado. Foram utilizados memorandos e diagramas que continham os produtos da análise. Na última etapa, codificação seletiva, definiu-se a categoria central que integrou todas as outras. Deste processo, foi construído o modelo teórico que representa o tema central do estudo. Para sua validação, foi apresentado a três participantes, com maior tempo de experiência como cuidadores, que foram capazes de se reconhecer no modelo exposto. Ao fundamentar esta investigação, no referencial do Interacionismo Simbólico, seguiu-se o pressuposto de que as ações do cuidador são significativas tanto para ele, quanto para o seu familiar, estabelecendo, dessa forma, as condições possíveis a uma interação simbólica, produtora de ações e comportamentos, que apenas podem ser acessadas, com base em uma análise que não desconsidere o processo de construção compartilhada de significados.

O Estudo foi aprovado pelo Comitê de Ética e Pesquisa da Universidade Federal de Alfenas sob o Parecer no 1.092.501. Os participantes foram orientados quanto aos objetivos da pesquisa e concederam sua anuência por meio da assinatura do Termo de Consentimento Livre e Esclarecido (TCLE). Foi-lhes garantido o anonimato, o sigilo e seus nomes foram substituídos por nomes fictícios.

\section{RESULTADOS E DISCUSSÃO}

Dos 20 participantes, 17 eram mulheres e três homens, com predomínio da faixa etária de 40 a 59 anos, com ensino médio, católicos, casados e com filhos. Quanto à ocupação anterior e atual, predominou a do lar, seguida da profissão de Professor e Diarista, com renda familiar de dois salários mínimos (considerou-se o salário mínimo vigente no valor de $\mathrm{R} \$ \mathbf{8 8 0 , 0 0 )}$ e ausência de renda pessoal. Em relação ao parentesco com o adoecido, houve o predomínio de filhos que assumiram o cuidado, seguido por esposas, irmãos, netos e sobrinhos. O tempo como cuidador compreendeu um intervalo entre sete meses a dez anos.

O modelo conceitual resultante da análise pôde ser representado pela categoria central: Ressignificando a vida e o papel de cuidador e as categorias e subcategorias, conforme Figura1.

Figura 1 - Modelo Teórico: Ressignificando a vida e o papel de cuidador

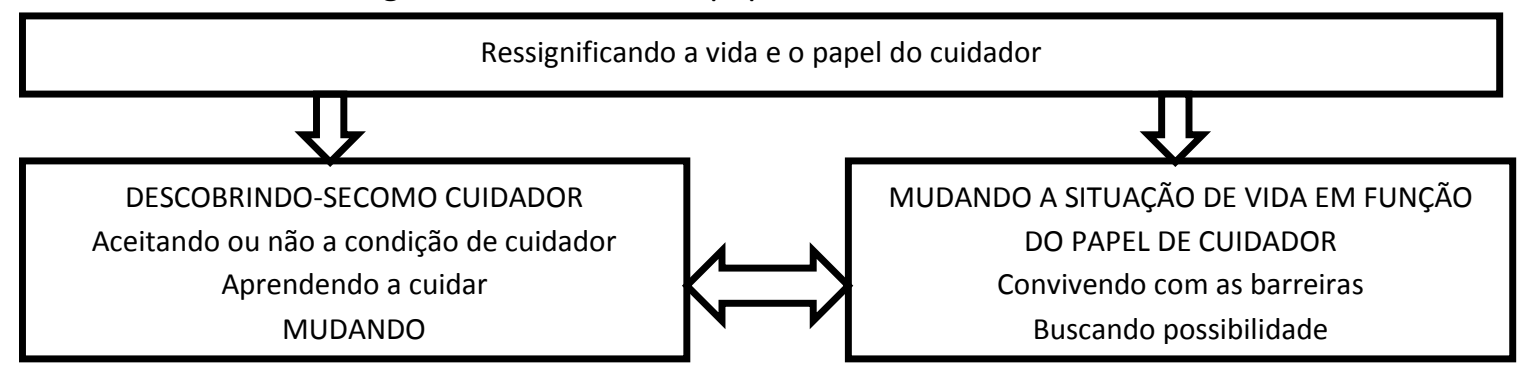

Fonte: Elaborado pela autora

\section{Descobrindo-se como cuidador}

Pôde-se apreender que o cuidador se descobre neste trabalho, e, baseado nessa descoberta, constrói um significado a esta função, porém nem sempre consegue discernir, com clareza, o momento em que passou a 
assumir o cuidado. Geralmente, esse fato, ocorre, involuntariamente, pelas conjunturas da enfermidade, uma vez que o adoecido encontrase em estado frágil e de vulnerabilidade. Alguns elementos favoreceram que a pessoa assuma a ação de cuidar, como: ser mulher, a proximidade geográfica e a dependência financeira. "[...] Desde que separei do meu companheiro, eu vim morar com a minha mãe, porque não tinha para onde ir e, quando ela ficou doente, eu que virei cuidadora dela, porque não trabalho, o meu irmão trabalha o dia todo [...]" (Paula, 52 anos). "[...] Quando o meu marido e o meu pai morreram, eu que tive que vir cuidar dela, porque, como sou mulher, acaba que sempre nós que temos que cuidar[...]" (Leticia, 58 anos).

Embora haja um número superior de mulheres, como cuidadoras, alguns homens assumiram esse trabalho, pela falta de alguém que realizasse tal atividade. "[...] Cuidar dela é desafiador, é especial, ela é tudo pra mim e não sei como vai ser minha vida quando ela se for, mas enquanto ela estiver aqui comigo, eu vou cuidar dela, cuidar como se o amanhã não existisse, como se ela fosse eterna para mim e acho que todo mundo que passa por uma situação parecida deve parar para pensar mesmo, é melhor ter ela aqui comigo do que em qualquer outro lugar [...]" (Lúcio, 38 anos).

O processo de aceitar o papel de cuidador nem sempre ocorre de forma tranquila e espontânea. $O$ cuidador, ante a necessidade de cuidar de seu familiar, vivencia muitos dilemas. Por ser uma tarefa árdua, o cuidador demonstra dificuldade em acolher uma situação que não seja passageira e parece perdurar por toda a vida do adoecido. "[...] Eu não aceito a situação que ele está agora, eu fico de mãos atadas e, isso para mim, é o fim do mundo, eu cuido dele, mas é muito difícil para mim[...]" (Luana, 54 anos).

Aprender a cuidar do seu familiar é uma tarefa permeada por acertos e erros, visto que o cuidador familiar desconhece a doença e não possui preparo que o capacite a exercê-la fazendo com que ele aprenda a cuidar, baseado na observação e na interpretação das manifestações. "[...] De tanto ver ela passar mal, agora eu até já sei quando as coisas vão ficar complicadas. Ela começa a puxar o ar para tentar respirar e logo vai ficando com a boca roxa, me dá pena ver ela nesta situação. Ela fala que a boca está seca e tem a sensação que vai morrer. Às vezes, só de colocar o oxigênio, ela já melhora, em outras vezes, tenho que levar para o hospital [...] (Amanda, 53 anos).

Percebe-se que o cuidador, na maioria das vezes, não entende a doença, ele sabe que é uma condição que afeta o funcionamento dos pulmões, que restringem as atividades diárias de seu familiar e exige a dependência da oxigenoterapia domiciliar para sobreviver. Inicialmente a experiência com a oxigenoterapia gera medo e insegurança ao cuidador, pois ele teme que algum problema aconteça com o concentrador de oxigênio. "[...] quando o meu marido começou usar o aparelho de oxigênio, foi muito complicado, porque eu tinha que ficar vigiando o tempo todo e eu não sabia o que fazer se acontecesse alguma coisa. Ele que me ajudava a vigiar se estava tudo certo [...]" (Ana, 80 anos).

Muda-se a situação de vida em função do papel de cuidador.

Muitas são as barreiras que o cuidador tem que enfrentar, diariamente, tornando-se fragilizado e vulnerável pelo constante estresse e tensão. O cuidador percebe que cuidar exige tempo e dedicação e que, por vezes, suas necessidades não podem ser atendidas, pois entende que as demandas do adoecido devem ser priorizadas. "[...] Fazia tempo que eu estava sentindo dor nos meus joelhos, mas não tinha tempo para ir ao médico até que comecei a mancar e sentir dor. Ele disse que é artrose e que eu não posso ficar o dia inteiro na lida [...]" (Cláudia, 81 anos).

Observam-se mudanças em sua vida, como o comprometimento da vida conjugal, podem levar o cuidador a demonstrar intenso sofrimento emocional. "[...] É difícil ficar longe do meu marido, morar em cidade diferente dele para ficar com minha avó. Embora o meu marido me dê o maior apoio, no fundo, eu tenho medo dele não aguentar esta situação e pedir a separação. Fico muito triste, mas também fico dividida com esta situação [...] (Raquel, 38 anos).

As dificuldades também se manifestam em questões básicas dos afazeres domésticos e da vida pessoal. O fato do adoecido não poder ficar sozinho em domicílio e a falta de apoio de outros membros da família contribuem, para que o cuidador perceba que não pode mais controlar a sua própria vida, o que corrobora para o seu isolamento social. "[...] Eu sempre gostei muito de sair, de ir ao barzinho com os meus amigos, mas agora fica difícil, porque toda vez que quero ou preciso sair, tenho que arrumar alguém para ficar com a minha mãe e, mesmo arrumando, a 
cabeça fica sempre quente, porque não sei o que está acontecendo aqui em casa. Eu até saio de vez em quando, mas não é mais como era antes [...]" (Leticia, 58 anos).

Além dos aspectos apontados, o custo adicional com a energia elétrica, também, representou um fator gerador de estresse, por constituir um acréscimo à despesa quanto à reduzida renda familiar. "[...] A conta de energia elétrica ficou muito cara, depois que ela começou a usar oxigênio o tempo todo. Eu já tentei conseguir desconto na Cemig (empresa de energia elétrica de Minas Gerais) e não dá certo, e eu fico perdendo tempo de ir lá na Cemig [...]" (Marina, 25 anos).

Para se adaptar à nova situação, o cuidador busca estratégias, como o apoio de vizinhos e da religião. [...] A minha vizinha é como se fosse alguém da minha família. Posso contar com ela para tudo que ela me ajuda [...]" (Cláudia, 81 anos). "[...] Cuidar dele é algo que vem de Deus mesmo, ele que me dá forças, porque se fossem as minhas, ia ser muito difícil, então hoje vivemos bem [...]" (Sara, 41 anos).

Dessa análise, de acordo com o referencial proposto, apreende-se que assumir os cuidados do adoecido com DPOC exige reestruturação da dinâmica à vida do cuidador familiar, tendo em vista que é uma pessoa da própria família que assume a responsabilidade pelo cuidado de seu doente. Ante as limitações ocasionadas pela doença, não recebe renumeração pelos seus serviços $^{(10-11)}$.

Os resultados apontam que a maioria das pessoas que assume o papel de cuidador familiar é mulher, em consonância à literatura ${ }^{(10-13)}$, porque se espera que a mulher assuma os cuidados com o adoecido, como uma extensão do sua função maternal. Traz, ainda, contribuição importante ao identificar o homem como cuidador. Embora a maioria esteja em uma faixa etária, economicamente produtiva, não possuem vínculo empregatício anterior e nem posterior, ao assumir o papel de cuidador de seu familiar, evidenciando a dependência financeira. Contudo o cuidado realizado pelos cuidadores informais pode ter benefícios, como o aumento do vínculo com a pessoa cuidada e um grande valor econômico, para a redução dos custos do cuidado, quando comparado aos custos da pessoa hospitalizada ${ }^{(10,14)}$.

Outros fatores podem ter contribuído para que alguém se torne cuidador, como o vínculo, a proximidade, a corresidência com a pessoa adoecida, as condições financeiras desfavoráveis, a falta de vínculo empregatício e a separação conjugal $^{(11)}$.

A DPOC é considerada uma patologia limitante que aumenta a dependência do adoecido e o obriga a utilizar dispositivos, para suporte respiratório com os quais o cuidador familiar não está preparado para lidar, aumentando as dificuldades para realizar os cuidados $^{(15)}$.

Observou-se que o cuidador se vê obrigado a lidar com elementos objetivos (concentrador de oxigênio e todos os seus acessórios) e subjetivos (concepções a respeito da DPOC e relações estabelecidas com a atividade de cuidado), relacionados ao novo papel que desempenha. As atividades efetuadas pelo cuidador, no cotidiano, levam-no a dedicar-se, cada vez mais tempo ao adoecido, originando sobrecarga, privações, isolamento social e as dificuldades financeiras que são situações geradoras de estresse e trazem intensa angústia, impotência e desesperança, em relação ao futuro e podem acarretar em sérios problemas de saúde à vida do cuidador ${ }^{(16)}$.

Estudo realizado com cuidadores, que convivem com os seus familiares e utilizam o concentrador de oxigênio, apontou que eles têm a sensação de que o dispositivo invadiu a sua casa, pois ele se vê cercado por fios e pelo barulho que é perturbador durante o sono ${ }^{(15)}$.

Verifica-se que 0 processo de reorganização dos papéis do cuidador familiar não transcorre de uma forma tranquila e natural, uma vez que não é planejado ou escolhido ${ }^{(17)} \mathrm{e}$, geralmente, tem início pela diminuição ou incapacidade da pessoa que adoeceu de prover os seus próprios cuidados ${ }^{(18)}$.

A condição de cuidador desperta um misto de sentimentos negativos e positivos relacionados ao fato de o cuidado tornar-se cada vez mais complexo pela severidade da sintomatologia, pela perda da independência do adoecido e pelo afeto entre a pessoa adoecida ${ }^{(19)}$. Entende-se, assim, que o processo de aceitar o papel de cuidador é complexo e intrinsicamente relacionado à elaboração de significados estabelecidos na interação. É nessa experiência que o cuidador sente o peso do que é cuidar e que não está preparado para lidar com essa condição ${ }^{(20)}$.

Nesse processo, o cuidador pode decodificar esse papel de interpretar a sintomatologia apresentada pelo adoecido e de avaliar a necessidade ou não de administração de 
oxigênio, o que pode contribuir, para que se sinta mais confiante e seguro, por conseguir cuidar acertadamente de seu familiar ${ }^{(20)}$. Esse processo é dinâmico, tendo em vista que a atribuição de sentido se modifica na interação ${ }^{(6,8)}$.

Esse fator é relevante, quando se considera que o cuidador familiar de pessoas, com doenças incapacitantes, é mais afetado que outros cuidadores $^{(10-11,13)}$, o que corrobora com 0 aumento significativo do nível de estresse, pelas atribuições pertinentes ao cuidado.

No caso da DPOC, por ser uma condição crônica e incapacitante, afeta tanto a vida do adoecido quanto a vida do seu cuidador familiar, pois ele vivencia diariamente o agravamento da sintomatologia, as restrições e a relutância do adoecido a usar o oxigênio de acordo com as orientações médicas. Essa situação impõe, cada vez mais, maior dependência do adoecido e, consequentemente, maior sobrecarga do cuidador, o que demanda suporte emocional, prático e informativo dos profissionais de saúde (21).

Portanto a experiência de ser cuidador é marcada por sofrimento, em decorrência do adoecimento do familiar e das situações problemáticas que envolvem o cuidar.

A dificuldade, para cuidar de sua própria saúde, também, emerge como problema, por falta de tempo, principalmente, quando eles são os únicos responsáveis pelos cuidados, o que compromete o seu bem-estar e o expõe a constante risco de adoecimento ${ }^{(3,22)}$. O cuidador percebe que se dedicar, integralmente, à tarefa de cuidar impede que ele realize atividades que praticava antes, o que lhe traz prejuízos à sua vida familiar e conjugal ${ }^{(22)}$.Tal situação ainda se agrava pela falta de apoio da família, que compromete a qualidade do cuidado prestado e a vida do cuidador que se encontra, por vezes, sozinho ${ }^{(20)}$.

Embora tenham que conviver com inúmeras barreiras, os cuidadores foram capazes de buscar estratégias que Ihes permitiram lidar com as situações adversas, para enfrentar a sua rotina diária de assistência ao adoecido e aliviar o estresse $^{(23)}$. As estratégias de enfrentamento representam possibilidades concretas ao cuidador que necessita diminuir a sobrecarga, de modo a que a sua vida tenha mais qualidade ${ }^{(24)}$.

A fé emerge como fonte de energia e esperança, para manter o equilíbrio emocional, promover o bem-estar e melhoria da qualidade de vida, por se sentirem confortados e fortalecidos, uma vez que constitui uma maneira de experienciar sua conexão com o eu, com os outros e com o Sagrado ${ }^{(25)}$ e, assim, reduzir os sentimentos negativos da tarefa de cuidar ${ }^{(11)}$

Apreende-se que o cuidador, diante da complexidade do cuidado, requer a mobilização de múltiplos saberes e fazeres, tornando-se, a partir dessas experiências, pessoas mais tolerantes, pacientes e mais humanas. Essa vivência traz ao cuidador possibilidades de ressignificar as demandas de seu novo papel.

\section{CONCLUSÕES}

Ser cuidador informal de pessoas com DPOC é ter a vida transformada, em função daquele que necessita ser cuidado, vivendo em um contexto que nem sempre é favorável, pois é marcado pela enfermidade, dilemas, sofrimentos e restrições. Paradoxalmente, contrapõem-se aspectos positivos, relativos às estratégias de enfrentamento, como o apoio da família e da religião, mostrando-se capaz de se tornar sensível e de ofertar cuidados ao familiar adoecido, bem como a atribuição de um novo significado à essa experiência.

O recorte transversal e o cadastro desatualizado de pessoas com DPOC podem ser considerados limitações deste estudo. No entanto o modelo teórico construído está em consonância com a literatura.

Para a enfermagem, a compreensão desse processo traz contribuições, porque permite um olhar ampliado às necessidades do cuidador e à importância de implementar programas, para atendê-lo na perspectiva da integralidade.

São necessários estudos que possam analisar as estratégias de apoio aos cuidadores informais de pessoas com condições crônicas no processo de adaptação a esse função.

\section{REFERÊNCIAS}

1- Reis AJ, Alves C, Furtado S, Ferreira J, DrummondM, Robalo-Cordeiro C. COPD exacerbations: Management and hospital discharge. Pulmonology 2018;24(6):345-50. DOI: doi: 10.1016/j.pulmoe.2018.06.006

2- Polverino F, Celli B. The Challenge of controlling the COPD Epidemic: Unmet Needs. Am J Med. 2018; 131(9S):1-6. DOI: 10.1016/j.amjmed.2018.05.001

3- Nakken N, Janssen DJA, Van den Bogaart EHA, Wouters EFM, Franssen FME, Vercoulen J $\mathrm{H}$, et al. Informal caregivers of patients with COPD: Home 
sweet home? Eur Respir Rev. 2015;24:498-504. DOI: $10.1183 / 16000617.00010114$

4- Garbaccio JL, Tonaco LAB. Características e Dificuldades do cuidador informal na assistência ao idoso. J Res Fundam Care 2019;11(3):680-86. DOI: 10.9789/2175-5361.2019.v11i3.680-686

5- Singh S, Estefan A. Selecting a Grounded Theory approach for nursing research. Glob Qual Nurs Res. 2018;5: 1-9. DOI: 10.1177/23333936 18799571

6- Haguette TMF. Metodologias qualitativas na sociologia. Petrópolis: Vozes; 1990.

7- Strauss A, Corbin J. Pesquisa qualitativa: técnicas e procedimentos para o desenvolvimento de teoria fundamentada. $2 a$ ed. Porto Alegre (RS): Artmed; 2008.

8- Blumer H. A natureza do Interacionismo simbólico. In: Morteuseu CD. Teoria de comunicação: Textos básicos. São Paulo: Mosaico; 1980.

9- Oliveira APA, Caldana RHL. As repercussões do cuidado na vida do cuidador familiar do idoso com demência de Alzheimer. Saude Soc. 2012; 21(3):675-85. DOI: 10.1590/S0104-12902012 000300013

10- Edwards VJ, Bouldin ED, Taylor CA, Olivari BS, McGuire LC. Characteristics and health status of informal Unpaid Caregivers - 44 States, District of Columbia, and Puerto Rico, 2015-2017. MMWR Morb Mortal Wkly Rep. 2020;69(7):183-88. DOI: 10.15585/mmwr.mm6907a2

11- Carbó GFC, García-Orellán R. Burden and Gender inequalities around Informal Care. Invest Educ Enferm. 2020;38(1):e.05. DOI: 10.17533/udea.iee.v38n1e10

12- Nunes SFL, Alvarez AM, Costa MFBNA, Valcarenghi RV. Fatores determinantes na transição situacional de familiares cuidadores de idosos com doença de parkinson. Texto Contexto - Enferm. 2019;28:e20170438. DOI: 10.1590/ 1980-265x-tce-2017-0438

13- Shah RJ, Collard HR, Morisset J. Burden, resilience and coping in caregivers of patients with interstitial lung disease. Heart Lung 2018; 47(3):264-68. DOI: 10.1016/j.hrtlng.2018.03.004 14- Alwin J, Karlson BW, Husberg M, Carlsson P, Ekerstad N. Societal costs of informal care of community-dwelling frail elderly people.Scand J Public Health. [Internet]. 2019;11(1):123-35. DOI: 10.1177/1403494819844354

15- Graney BA, Wamboldt FS, Baird S, Churney T, Churney $\mathrm{T}$, Fier $\mathrm{K}$, et al. Informal caregivers experience of supplemental oxygen in pulmonary fibrosis. Health Qual Life Outcomes 2017;15 (133):1-6. DOI: 10.1186/s12955-017-0710-0

16- Vasconcelos AO, Batista VC, Back IR, Miguel MEGB, Marquete VF, Marcon SS, et al. Avaliação da resiliência de pessoas com condições crônicas e cuidadores. Rev Enferm UFPE;13(3):690-6. DOI: 10.5205/1981-8963-v13i03a239188p690-6962019

17- Pereira S, Duque E. El cuidado de las personas mayores dependientes. La sobrecarga de los cuidadores. Rev Kairós 2017 [citado em 24 nov 2019]; 20(1):187-202. Available in: https://pesquisa.bvsalud.org/portal/resource/pt/ biblio-881102?lang=en

18- Leslie M, Gray PR, Eales J, Fast J, Magnaye A, Khayatzadeh-Mahani $A$. The care capacity goals of family carers and the role of technology in achieving them. BMC Geriatr. 2020;20(52):1-12. DOI: $10.1186 / \mathrm{s} 12877-020-1455-x$

19- Landeiro MJL, Peres HHC, Martins T. Avaliação de necessidades informacionais dos cuidadores domiciliares. Rev Enferm UFSM 2015; 5(3):486-98. DOI: 10.5902/2179769216886

20- Kokorelias KM, Lu FKT, Santos JR, Xu Y, Leung $\mathrm{R}$, Cameron $\mathrm{Jl}$. Caregiving is a full-time job impacting stroke caregivers health and wellbeing: A qualitative meta-synthesis. Health Soc Care Community 2020;28(2):325-40. DOI: 10.1111/hsc. 12895

21- Strang S, Fährn J, Strang P, Ronstad A, Danielsson L. Support to informal caregivers of patients with severe chronic obstructive pulmonary disease: a qualitative study of caregivers and professionals experiences in Swedish hospitals. BMJ Open 2019;9(8):e028720. DOI: 10.1136/bmjopen-2018-028720

22- Bhan N, Rao N, Raj A. Gender differences in the associations between informal caregiving and wellbeing in low- and middle-income countries. J Womens Health 2020;1-11. DOI: 10.1089/jwh. 2019.7769

23- AldanaMSC, Ninõ EYD, Carvajal RR, Esteban ANP, Gonzales GMC. Sobrecarga y apoyos en el cuidador familiar de pacientes con enfermedad crónica. Rev Cuid. 2019;10(3): e649. DOI: 10.15649/cuidarte

24- Magalhães JF, Lopes RE, Nóbrega-Therrien SM, Vasconcelos SB. Caregiver women's coping strategies toward schizophrenia bearing people. J Res Fundam Care 2018;10(3):793-800. DOI: 10.9789/2175-5361.rpcfo.v10.6206 
25- Gergianaki I, Kampouraki M, Williams S, Tsiligiannil IG. Assessing spirituality: Is there a beneficial role in the management of COPD?NPJ Prim Care Respir Med 2019;29(1): 1-5. DOI: 10.1038/s41533-019-0134-x

Nota: Este estudo é um recorte da dissertação de mestrado intitulada "O significado de ser cuidador de pessoa com doença pulmonar obstrutiva crônica dependente de oxigenoterapia domiciliar" para obtenção do título de mestre pelo Programa de Pós-graduação em Enfermagem da Universidade Federal de Alfenas-MG.

"O presente trabalho foi realizado com apoio da Coordenação de Aperfeiçoamento de Pessoal de Nível Superior - Brasil (CAPES) - Código de Financiamento 001"

Recebido em: 01/12/2019

Aprovado em: 08/04/2020

Endereço de Correspondência:

Silvana Maria Coelho Leite Fava

R. Gabriel Monteiro da Silva, 700 - Centro

Alfenas-MG

CEP: 371130-001

silvana.fava@unifal-mg.edu.br 